\title{
Modelo de aprendizaje cooperativo del idioma inglés utilizando tecnologías de la información y comunicación
}

\begin{abstract}
(c) (i) (-)(2)
Cooperative learning model of the English language using information and communication technologies
\end{abstract}

Nanci Margarita Inca Chunata. ${ }^{1}$, Ana Gabriela Reinoso Espinosa. ${ }^{2}$, Evelyn Carolina Macias Silva. ${ }^{3} \&$ Sandra Elizabeth Merino Hernández. ${ }^{4}$

\section{Abstract.}

DOI: https://doi.org/10.33262/concienciadigital.v3i2.1208

The objective of this article is to reveal the impact of a combined cooperative learning strategy and information and communication technologies to improve English language learning. The study population was of 100 students: 50 from the intervention group and 50 from the control group, who belonged to the Chemical Engineering degree at the Higher Polytechnic School of Chimborazo. Through a quasi-experimental analysis and control group, the development of the four language skills such as listening, reading, speaking, writing, and the two linguistic areas: grammar and vocabulary. The statistically analyzed results show that the students improved their skills in the English language proficiency. on geometric elements to reason about conditional probability. The productions analyzed before and after the instruction. The purpose was to contribute to the construction of the meaning of the formula programmed in the software or firmware of a network interface, by promoting the ability to carry out the cognitive construction of the communication channel and its

1 Escuela Superior Politécnica de Chimborazo, Facultad de Ciencias. Riobamba, Ecuador. nanci.inca@espoch.edu.ec

2 Escuela Superior Politécnica de Chimborazo, Facultad de Ciencias, Riobamba, Ecuador. ana.reinoso@espoch.edu.ec

3 Escuela Superior Politécnica de Chimborazo, Facultad Ciencias. Riobamba, Ecuador. evelyn.macias@espoch.edu.ec

4 Unidad Educativa Pedro Vicente Maldonado. Riobamba - Ecuador, sandramerino37@ yahoo.com 
behavior in a work setting. Students who developed this skill showed less difficulty in their resolutions and came up with new ideas on optimizing.

Keywords: English Language, Collaborative Learning, Information and Communication Technologies

\section{Resumen.}

El objetivo de este artículo es revelar el impacto de una estrategia combinada de aprendizaje cooperativo y las tecnologías de la información y comunicación para mejorar el aprendizaje del idioma inglés. La población de estudio la conformaron 100 estudiantes: 50 del grupo de intervención y 50 de control, pertenecientes a la carrera de ingeniería Química de la Escuela Superior Politécnica de Chimborazo. Por medio de un análisis cuasi experimental y grupo control, se conoció el desarrollo de las cuatro destrezas de la lengua: escuchar, leer, hablar y escribir y las dos áreas lingüísticas: gramática y vocabulario. Los resultados analizados estadísticamente evidencian que los estudiantes mejoraron sus habilidades en el dominio del idioma inglés.

Palabras claves: Idioma Inglés, Aprendizaje colaborativo, Tecnologías de la Información y Comunicación

\section{Introducción.}

El Aprendizaje Cooperativo es el conjunto de procedimientos de enseñanza que parten de la organización de la clase en pequeños grupos mixtos y heterogéneos donde los estudiantes trabajan conjuntamente, de forma coordinada entre sí, para desarrollar las 4 destrezas del idioma (Listening, Speaking, Reading, Writing) y profundizar en su propio aprendizaje. Por tanto, requiere de la participación directa y activa del alumnado.

En el proceso de enseñanza-aprendizaje suelen distinguirse tres sistemas de motivación del estudiante para el aprendizaje, y de organización por parte del profesor, que vienen determinados por el valor que se le atribuye a alcanzar determinadas metas y por el tipo de interacción que se establece entre los alumnos. Son el sistema individualista, el sistema competitivo y el sistema cooperativo (Barkley \& Cross, 2007).

En una situación cooperativa, los individuos procuran obtener resultados que sean beneficiosos para ellos mismos y para todos los demás miembros del grupo. El aprendizaje cooperativo es el empleo didáctico de grupos reducidos en los que los alumnos trabajan juntos para maximizar su propio aprendizaje y el de los demás. Este método contrasta con el aprendizaje competitivo, en el que cada alumno trabaja en contra de los demás para alcanzar un solo objetivo la calificación de "10" que sólo uno o algunos pueden obtener (Johnson D. W., 1999). Con el aprendizaje individualista, en el que los estudiantes trabajan por su cuenta para lograr metas de aprendizaje desvinculadas de las de los demás alumnos. 
En el aprendizaje cooperativo y en el individualista, los maestros evalúan el trabajo de los alumnos de acuerdo con determinados criterios, en el aprendizaje competitivo, los alumnos son calificados según una cierta norma o rendimiento. Tanto aprendizaje competitivo e individualista presentan limitaciones respecto de cuándo y cómo emplearlos en forma apropiada, el docente de Inglés puede organizar cooperativamente cualquier tarea didáctica. (Lin, 2008)

De los tres sistemas de motivación del alumno para el aprendizaje y de organización de la docencia por parte del profesor el que aporta mejores resultados a los alumnos es el sistema cooperativo (Kagan, 1994). El sistema cooperativo produce mejores rendimientos que el sistema individualista y que el sistema competitivo, el sistema cooperativo - aun compitiendo con otros grupos de compañeros- es superior al sistema competitivo, y el sistema cooperativo -sin competir con otros grupos de compañeros-es superior al sistema cooperativo compitiendo con grupos de compañeros (Torrego, 2012).

Tabla 1 Características de los sistemas de enseñanza-aprendizaje

\begin{tabular}{|c|c|c|c|}
\hline Características & Individualista & Competitivo & Cooperativo \\
\hline $\begin{array}{l}\text { Las metas que se } \\
\text { Proponen }\end{array}$ & $\begin{array}{l}\text { 1. Propio aprendizaje } \\
\text { 2. Aceptación social }\end{array}$ & Ser el mejor & $\begin{array}{l}\text { 1.Conseguir la misma } \\
\text { meta } \\
\text { 2. Contribuir al logro } \\
\text { grupal }\end{array}$ \\
\hline Estructura de la meta & $\begin{array}{l}\text { El estudiante alcanza } \\
\text { sus metas con } \\
\text { independencia }\end{array}$ & $\begin{array}{l}\text { El estudiante alcanza } \\
\text { sus metas sólo. }\end{array}$ & $\begin{array}{l}\text { El alumno alcanza sus } \\
\text { metas sólo si los } \\
\text { compañeros del grupo } \\
\text { las alcanzan }\end{array}$ \\
\hline $\begin{array}{l}\text { Las atribuciones que } \\
\text { hacen de su éxito }\end{array}$ & $\begin{array}{l}\text { 1. Esfuerzo } \\
\text { 2. Habilidad personal }\end{array}$ & $\begin{array}{l}\text { Destrezas superiores } \\
\text { a los demás }\end{array}$ & $\begin{array}{l}\text { 1. Esfuerzo propio } \\
\text { 2. Esfuerzo del grupo }\end{array}$ \\
\hline $\begin{array}{l}\text { Interacción con los } \\
\text { Compañeros }\end{array}$ & No existe interacción & Interacción negativa & Interacción positiva \\
\hline $\begin{array}{l}\text { Cómo son los } \\
\text { compañeros para mí }\end{array}$ & Indiferentes & Rivales & Colaboradores \\
\hline $\begin{array}{l}\text { Correlación entre mi } \\
\text { meta y la del grupo }\end{array}$ & Sin correlación & Correlación negativa & Correlación positiva \\
\hline $\begin{array}{l}\text { Cómo es la } \\
\text { recompensa por la } \\
\text { tarea }\end{array}$ & Sólo individual & Individual y no grupal & Individual y grupal \\
\hline
\end{tabular}

El aprendizaje cooperativo comprende tres tipos de grupos de aprendizaje:

Los equipos de base cooperativos tienen un funcionamiento de largo plazo (por lo menos de casi un año) y son grupos de aprendizaje heterogéneos, con miembros permanentes, cuyo principal objetivo es posibilitar que sus integrantes se brinden unos a otros el apoyo, la ayuda, 
el aliento y el respaldo que cada uno de ellos necesita para tener un buen rendimiento. Los grupos de base permiten que los alumnos entablen relaciones responsables y duraderas que los motivarán a esforzarse en sus tareas, a progresar en el cumplimiento de sus obligaciones escolares (como asistir a clase, completar todas las tareas asignadas, aprender) y a tener un buen desarrollo cognitivo y social (Zhang, 2010).

Los grupos formales de aprendizaje cooperativo funcionan durante un período que va de una hora a varias semanas de clase. En estos grupos, los estudiantes trabajan juntos para lograr objetivos comunes, asegurándose de que ellos mismos y sus compañeros de grupo completen la tarea de aprendizaje asignada. Este tipo de grupos son aplicables para cada clase de ingleses enfocados a desarrollar una destreza específica. Cuando se emplean grupos formales de aprendizaje cooperativo, el docente debe:

a. especificar los objetivos de la clase,

$b$. tomar una serie de decisiones previas a la enseñanza,

c. explicar la tarea y la interdependencia positiva a los alumnos,

$d$. supervisar el aprendizaje de los alumnos e intervenir en los grupos para brindar apoyo en la tarea o para mejorar el desempeño interpersonal y grupal de los alumnos, y

$e$. evaluar el aprendizaje de los estudiantes y ayudarlos a determinar el nivel de eficacia con que funcionó su grupo.

Los grupos formales de aprendizaje cooperativo garantizan la participación activa de todos los alumnos en las tareas intelectuales de organizar el material, explicarlo, resumirlo e integrarlo a las estructuras conceptuales existentes.

Los equipos informales de aprendizaje cooperativo operan durante unos pocos minutos hasta una hora de clase. El docente puede utilizarlos para el desarrollo una actividad de enseñanza directa. La actividad de estos grupos informales suele consistir en una charla de tres a cinco minutos entre los estudiantes antes y después de una clase, o en diálogos de dos a tres minutos entre pares de estudiantes durante el transcurso de una clase magistral. Al igual que los grupos formales de aprendizaje cooperativo, los grupos informales le sirven al maestro para asegurarse de que los alumnos efectúen el trabajo intelectual de organizar, explicar, resumir e integrar el material a las estructuras conceptuales existentes durante las actividades de enseñanza directa. (Torrego, 2012)

Analizado el aprendizaje cooperativo, sus ventajas y desventajas, es importante poner énfasis en el aprendizaje cooperativo para ayudar a los estudiantes a aprender una lengua extranjera.

(Ur, 2005) expone que el trabajo cooperativo ayuda a fomentar a que los estudiantes desarrollen habilidades comunicativas, lo cual es esencial al momento de aprender una lengua diferente a la materna.

Penny (Ur, 2005) señala que los estudiantes adquieren el idioma mediante comunicación significativa, es decir, en el proceso de interacción negociación y transmisión de significados 
en situaciones intencionales. Esta interacción significativa puede tener lugar entre dos o más estudiantes que desarrollan el conocimiento del sistema lingüístico como resultado del intercambio y de la negociación de los significados.

El aprendizaje cooperativo es una herramienta facilitadora del aprendizaje y adquisición del conocimiento en el idioma Inglés como lengua extranjera, debido a que entre pares, los estudiantes están expuestos a inputs comprensibles (Yule, 2014). La relevancia que tienen los métodos de enseñanza en el aprendizaje de una lengua extranjera. Además, otro punto importante, es la preparación para grupos cooperativos; es decir, los estudiantes tienen que estar conscientes del rol que cada uno tiene, para así evitar lo ya antes mencionado, donde solo unos pocos realizan el trabajo.

Por el otro lado, Jhonnson menciona un método que permite a los estudiantes desarrollar sus 23 habilidades en escritura y comprensión lectora de manera más eficiente, el cual se integra la lectura y Composición cooperativa (Cooperative Integrated Reading and Composition). (Johnson D. , 1975). Este modelo permite que los estudiantes se ayuden entre ellos sobre lo que ya han leído. También, anima a los estudiantes a leer en voz alta, lo cual según otra investigación (Lin, 2008) puede tener un gran impacto en el desarrollo de sus habilidades. Las actividades del modelo de Integración de lectura y composición cooperativa, tiende a focalizarse en la estructura del lenguaje, esto es, vocabulario y gramática.

El aprendizaje cooperativo hace cuatro diferentes aportaciones para el docente y estudiantes en el aprendizaje de una lengua extranjera, de las cuales se nombran tres, que fueron consideradas como las más importantes en este estudio.

En un primer lugar el aprendizaje cooperativo en una lengua extranjera puede ayudar al profesor a tener una concepción más humanista, es decir, entender a los estudiantes capaces de aprender en grupo, favoreciendo la participación de ellos y permitiéndoles sentirse en un ambiente cómodo y próspero en el aula.

En segundo lugar, el aprendizaje cooperativo de la lengua aporta al momento de hacer del aprendizaje cooperativo el eje de clase, pero también con diversas aportaciones del aprendizaje individual, enfocándose en la interacción y comunicación entre pares lo cual ayuda a la adquisición individual de la lengua.

En tercer lugar, el aprendizaje cooperativo permite la permeabilidad y aplicabilidad de recursos prácticos, lo cual se refiere a que los recursos del aula de clases ya sean: computadores, pizarra, etc. para que el profesor los aplique en el aprendizaje cooperativo de una lengua extranjera.

Es importante que en las clases de una lengua extranjera practiquen en el tiempo de duración de la misma. En un aprendizaje tradicional, el profesor es el que habla la mayoría del tiempo, no dejando espacio para que el alumno practique la lengua enseñada. Además, según (Oxford 
University Press 2011), el estudiante no tiene otra oportunidad de hablar en ese idioma en su vida diaria, por esta razón, es muy relevante que los estudiantes practiquen la habilidad de hablar en el idioma dentro del aula. Más aún, a través del aprendizaje cooperativo, el estudiante tendrá que discutir, opinar y todo lo que involucra ponerse de acuerdo en un grupo en el idioma que se está aprendiendo.

Por consiguiente, el estudiante pondrá en práctica su habilidad de hablar en el idioma extranjero de forma activa dentro del aula. En general, el área de la enseñanza de la lengua ha experimentado grandes cambios en comparación a años anteriores (Zhang, 2010).

Históricamente, la enseñanza tradicional estaba centrada en el profesor, donde los métodos destacados para la enseñanza y aprendizaje, fueron el método Gramática-Traducción (Grammar translation Method) y el método Audiolingual (Audio-lingual Method). Mediante estos métodos, los estudiantes requerían estar concentrados en ciertos aspectos del lenguaje, sin tener una adecuada práctica para su desarrollo y aplicación del aprendizaje.

Es por el aquello, (Zhang, 2010) concluye que la enseñanza, al ver evidencias sobre estos métodos, estaba plenamente basada en reglas de memorización para entender la morfología y la sintaxis de la lengua extranjera. Otro aspecto relevante que resalta este autor, es la pobre comunicación entre los alumnos dentro de la clase, ya que la interacción era completamente profesor-alumno y la interacción entre estudiante-estudiante era mínima. Esto se debía a que los estudiantes eran vistos como aprendices buscando la adquisición del conocimiento, más que desarrollar habilidades comunicativas.

Dentro de la enseñanza de las lenguas, se puede notar como el Aprendizaje Cooperativo el estimular la interacción comunicativa y crear oportunidades "reales" de escuchar y hablar.

Las estrategias de aprendizaje cooperativo y TIC se conciben en esta investigación como el modelo que puede envolver al estudiante en la construcción de un andamiaje sólido y en el análisis de la información para adquirir el conocimiento, lo que McKenzie (2000) llama un "scaffolded approach to concept building"; y promover la interacción auténtica en la que los estudiantes pueden practicar las formas del idioma extranjero, expresando ideas, sentimientos y emociones como en la vida real. Por otra parte, las explicaciones que se originan entre unos y otros, puede llevarlos a un entendimiento más profundo en el uso de una lengua extranjera y, por ende, al desarrollo de la competencia comunicativa (Brown, 2014).

Un conjunto de autores ha ahondado en los procesos cooperativos que favorecen el aprendizaje. Durán y Monereo (2012) destacan las estrategias Tutoría entre iguales, Tareas de rompecabezas, Aprendiendo juntos y Grupos de investigación que, sin ser las únicas existentes, probablemente son las más usadas, aquellas que nos han permitido hacer adaptaciones para diseñar actividades en la virtualidad y crear el andamiaje necesario para el desarrollo de habilidades de la lengua en cada lección. 
Johnson y Johnson (2003) señalan que cada estrategia sigue un proceso sistemático y bien estructurado, sin embargo, el docente debe asegurarse de que cada actividad contenga los principios cooperativos básicos que exija la participación de los miembros del grupo para lograr objetivos comunes.

La importancia de este estudio se asocia a la necesidad imperiosa de proporcionar un camino para innovar la práctica pedagógica, que permita dejar de lado la enseñanza rígida y memorística del idioma extranjero que aún persiste en los contextos educativos y se convierta en un ambiente de constante interacción, para que los estudiantes desarrollen la competencia comunicativa que les permitirá estar a la vanguardia de todos los procesos económicos, cultuales, tecnológicos, científicos y educativos presentes en el actual mundo global.

De acuerdo con los criterios vertidos; este estudio tiene el objetivo primordial de dar a conocer el impacto de una estrategia combinada de aprendizaje cooperativo a través de las TIC, que abra el camino hacia una metodología innovadora para mejorar el aprendizaje del idioma inglés; plantea un ambiente de interacción permanente en el que la solidaridad, la tolerancia, el respeto al criterio ajeno, la responsabilidad, colaboración y ayuda mutua a través de las tecnologías de la información y comunicación sean los pilares axiológicos de la clase. Las Tics son herramientas a través de las cuales se realiza la adquisición, producción,

síntesis, almacenamiento y comunicación de información, combinando texto, imagen, video y sonido.

Este trabajo contribuye a la investigación en el campo, en tanto proporciona una pauta para gestionar la educación en forma diferente. Los docentes contarán con un método de enseñanza aprendizaje que aporta significativamente para que los estudiantes mejoren el aprendizaje de la lengua extranjera.

\section{Metodología.}

En este trabajo se utilizó un diseño de investigación cuasi experimental pre-test, post-test y grupo control, para determinar la influencia de la metodología cooperativa utilizando las TIC en el aprendizaje del inglés.

Este tipo de diseño analiza la certeza de las hipótesis planteadas y permite la manipulación intencional de la variable independiente para ver los resultados que provoca y la medición de la variable dependiente.

Para efectos de la presente investigación se trabajó con una muestra de 100 estudiantes regulares en la carrera de Ingeniería Química, 50 estudiantes del grupo experimental, y 16 del grupo control, con edades comprendidas entre los 18 y 24 años. Los grupos se establecieron de acuerdo con la distribución de paralelos o grupos de trabajo. 
Se trabajaron dos variables. La independiente corresponde a la metodología cooperativa utilizando las tecnologías de la información y comunicación. Se refiere a una estrategia educativa altamente estructurada de trabajo en equipo, donde las actividades están diseñadas para que cada participante contribuya con su conocimiento para el logro de metas comunes. Esta variable se puso en operación en cuatro dimensiones: tutoría entre iguales, aprendizaje juntos, rompecabezas y grupos de investigación.

La variable dependiente: aprendizaje del idioma inglés, que se entiende como el desarrollo de la competencia comunicativa, habilidad para comprender y utilizar el inglés para expresar ideas, sentimientos y emociones de acuerdo con un contexto y situación social.

La investigación se enfocó en seis dimensiones: las cuatro destrezas de la lengua (listening, speaking, reading, writing) y las dos áreas lingüísticas (grammar y vocabulary); todas encaminadas a comprobar la comprensión general y en detalle de textos tanto escritos como orales, la habilidad para organizar oraciones y párrafos con cohesión y coherencia, la fluidez y exactitud en el logro del propósito comunicativo, el uso correcto de las estructuras gramaticales y la comprensión del significado.

Se realizó un proceso de intervención lingüística basado en la metodología cooperativa a través de las TIC, partiendo del diseñó del sílabo con los contenidos curriculares normados para la carrera. Siguiendo con el enfoque las cuatro dimensiones (estrategias cooperativas) señaladas, estas se crearon en el aula virtual diseñada en la plataforma Moodle, el sitio virtual de aprendizaje Google Drive y el correo electrónico, así como con el uso de podcasts. En cada lección hubo una fase introductoria en la que el docente realizó la presentación funcional de la lengua a ser aprendida y practicada en esa sesión.

Luego una fase de desarrollo del tema propuesto, en la que los equipos de trabajo desarrollaron las actividades cooperativas en la virtualidad.

Finalmente, los estudiantes de cada equipo en turnos, de acuerdo con los roles asignados en esa actividad, concluyeron la información en forma oral o escrita para la retroalimentación y consolidación del aprendizaje.

En el desarrollo de los procedimientos los estudiantes interactuaron activamente y perfeccionaron su competencia comunicativa utilizando las destrezas según la naturaleza del tema. Para una adecuada organización en el proceso de intervención, se planificó cada sesión, describiendo el indicador de logro, la secuencia metodológica, la estrategia cooperativa y el recurso tecnológico utilizado.

Por otro lado, los integrantes del grupo control, después del pre-test, trabajaron los mismos contenidos sobre la base de una metodología tradicional, sustentada en el enfoque estructuralista que apunta al tratamiento de la lengua como disciplina de estudio, utilizando 
un texto y un CD de audio de la bibliografía básica obligatoria para el curso. Su aprendizaje estuvo dirigido básicamente a aspectos gramaticales y estructurales.

Para medir la variable dependiente, aprendizaje de inglés, se utilizaron los siguientes instrumentos:

Pre-test o prueba de entrada. Al inicio del trabajo experimental se aplicó a cada participante de los grupos experimental y control una prueba de conocimiento, pre-test, consistente en preguntas que cubrían todas las destrezas: listening, reading, speaking y writing y las dos áreas de la lengua, grammar y vocabulary. La prueba fue validada con estándares estándares establecidos por el Marco Común Europeo de Referencia para las Lenguas: Aprendizaje, Enseñanza, Evaluación.

Post-test o prueba de salida. Una vez concluido el programa de capacitación, se aplicó una prueba de salida (post-test), de construcción paralela a la de entrada. Se evaluó a los estudiantes en función de los mismos criterios empleados para el pre-test; esto es, las destrezas y formas lingüísticas de la lengua. La prueba se elaboró con base en muestras de exámenes estandarizados de Cambridge Key English Test.

Las preguntas establecidas para el desarrollo de las destrezas de la lengua fueron estructuradas con ítems de completación, selección múltiple, verdadero/falso. La calificación se realizó de acuerdo con la escala de valoración y el índice correspondiente que se observa en la tabla 2.

Tabla 2. Categorías de calificación por rangos de notas

\begin{tabular}{cl}
\hline RANGO & VALORACIÓN \\
\hline $0-3$ & Insuficiente \\
$4-5$ & Regular \\
$6-7$ & Bueno \\
$8-9$ & Muy Bueno \\
10 & Sobresaliente \\
\hline
\end{tabular}

Cada examen registró seis secciones que cubrían todas las destrezas de la lengua y las dos áreas lingüísticas. Cada pregunta sobre las primeras estuvo constituida por dos partes con alternativas de respuesta de diferente naturaleza, ya sea para completar, de selección múltiple verdadero/falso y ensayo. Las preguntas sobre gramática y vocabulario contuvieron 20 ítems, con 3 y 4 alternativas de respuesta, respectivamente. El examen se administró en forma individual a los estudiantes con una duración aproximada de 90 minutos.

Con las evaluaciones de pre y post-test se verificó el desarrollo del aprendizaje del idioma inglés, a través del uso de la referida metodología, de acuerdo con las características de las hipótesis y los objetivos de la investigación, que fueron los siguientes: 
Hipótesis general: la metodología cooperativa utilizando las tecnologías de la información y comunicación mejora el aprendizaje del idioma inglés.

Para contrastar la hipótesis, y probar la relación existente entre las variables, se aplicó el paquete computacional SPSS y el estadístico $\mathrm{T}$ de Student. Por la naturaleza de la investigación se utilizó la estadística descriptiva, se empleó la distribución de frecuencias absolutas y porcentuales y se usó la diferencia porcentual como medida de incremento entre el pre y post-test. La verificación de las hipótesis se realizó a través de la prueba de diferencia de medias pareadas de los puntajes obtenidos en el pre y post-test.

\section{Resultados.}

En la Tabla 3 se evidencian los resultados obtenidos en el pre y pos-test del grupo experimental, por cada habilidad y área lingüística.

Tabla 3 Estadísticos descriptivos de las calificaciones grupo experimental

\begin{tabular}{lrrrrr}
\hline & $\mathrm{N}$ & Minimum & Maximum & Mean & Std. Deviation \\
\hline Número de Estudiantes & 50 & 1 & 16 & 8.78 & 4.769 \\
listening pre & 50 & 3 & 7 & 4.50 & 1.111 \\
listening post & 50 & 6 & 10 & 8.66 & 1.255 \\
Reading pre & 50 & 3 & 8 & 4.68 & 1.477 \\
Reading post & 50 & 5 & 9 & 7.88 & 1.365 \\
Speaking Pre & 50 & 3 & 6 & 3.94 & .913 \\
Speaking Post & 50 & 6 & 10 & 8.12 & .918 \\
Grammar pre & 50 & 2 & 6 & 3.86 & 1.050 \\
Grammar post & 50 & 6 & 9 & 7.80 & .881 \\
Vocabulary pre & 50 & 3 & 6 & 3.98 & .869 \\
Vocabulary post & 50 & 7 & 9 & 8.24 & .771 \\
Writing pre & 50 & 2 & 6 & 3.92 & 1.175 \\
Writing post & 50 & 5 & 10 & 8.26 & 1.242 \\
Valid N (listwise) & 50 & & & & \\
\hline
\end{tabular}

Notación: N número de estudiantes

Se puede observar que todas las habilidades se vieron más favorecidas con este programa de intervención: en el pre-test, 11 estudiantes obtuvieron una mejora significativa para el 
listening de 4.5 a 8.66, en Reading de 4.68 a 7.88, Speaking de 3.94 a 8.12, en Grammar de 3.86 a 7.80 , Vocabulary de 3.98 a 8.24 y Writening de 3.92 a 8.26

Tabla 4 Estadísticos descriptivos de las calificaciones grupo de control

\begin{tabular}{lccccc}
\hline & N & Minimum & Maximum & Mean & Std. Deviation \\
\hline Listening pre & 50 & 3 & 6 & 4.34 & 1.002 \\
Listening post & 50 & 4 & 6 & 5.04 & .755 \\
Reading pre & 50 & 3 & 8 & 4.68 & 1.151 \\
Reading post & 50 & 5 & 7 & 5.88 & .594 \\
Speaking Pre & 50 & 3 & 6 & 4.00 & .881 \\
Speaking Post & 50 & 4 & 7 & 4.86 & .857 \\
Grammar pre & 50 & 2 & 6 & 3.92 & 1.085 \\
Grammar post & 50 & 3 & 6 & 4.72 & .970 \\
Vocabulary pre & 50 & 3 & 7 & 3.94 & 1.132 \\
Vocabulary post & 50 & 4 & 6 & 5.22 & .679 \\
Writening pre & 50 & 3 & 5 & 3.92 & .752 \\
Writening post & 50 & 4 & 8 & 5.52 & .931 \\
Valid N (listwise) & 50 & & & & \\
\hline
\end{tabular}

Los resultados de la Tabla 4 resumen las calificaciones obtenidas por el grupo de control pres test y post test, estos datos permitirán verificar la hipótesis de manera cuantitativa.

Nivel de significancia: $\alpha=0.05$.

Regla de decisión: Rechace la hipótesis nula si p-valor $<0.05$.

Cálculos: se utilizó la prueba T para muestras independientes en el programa SPSS, cuyos resultados principales son los siguientes:

Tabla 5 Prueba de significancia

\begin{tabular}{lcccccc}
\hline & & \multicolumn{2}{c}{ Test Value $=0$} \\
& & & & Sig. (2-tailed) & $\begin{array}{c}\text { Mean } \\
\text { Difference }\end{array}$ & $\begin{array}{c}\text { 95\% Confidence Interval of } \\
\text { the Difference }\end{array}$ \\
\cline { 5 - 7 } & & & & & Lower & Upper \\
\hline Grupo & 29.850 & 99 & .000 & 1.500 & 1.40 & 1.60 \\
listening pre & 41.863 & 99 & .000 & 4.420 & 4.21 & 4.63 \\
listening post & 32.763 & 99 & .000 & 6.850 & 6.44 & 7.26 \\
Reading pre & 35.531 & 99 & .000 & 4.680 & 4.42 & 4.94 \\
Reading post & 47.401 & 99 & .000 & 6.880 & 6.59 & 7.17
\end{tabular}


ISSN: 2600-5859

www.concienciadigital.org

Vol. 3, N², p. 55-68, abril-junio, 2020

$\begin{array}{lllllll}\text { Speaking Pre } & 44.467 & 99 & .000 & 3.970 & 3.79 & 4.15 \\ \text { Speaking Post } & 34.868 & 99 & .000 & 6.490 & 6.12 & 6.86 \\ \text { Grammar pre } & 36.607 & 99 & .000 & 3.890 & 3.68 & 4.10 \\ \text { Grammar post } & 34.752 & 99 & .000 & 6.260 & 5.90 & 6.62 \\ \text { Vocabulary pre } & 39.433 & 99 & .000 & 3.960 & 3.76 & 4.16 \\ \text { Vocabulary } & 40.039 & 99 & .000 & 6.730 & 6.40 & 7.06 \\ \text { post } & 39.941 & 99 & .000 & 3.920 & 3.73 & 4.11 \\ \text { Writening pre } & 39.204 & 99 & .000 & 6.890 & 6.54 & 7.24 \\ \text { Writening post } & & & & & \end{array}$

En la Tabla 5 se observa la prueba de significancia de diferencia de medias para muestras independientes para el pos- test. La diferencia de medias de ambos grupos es superior a 1.5 para cada bloque de aprendizaje con un error estándar de la diferencia de 0.01 y por lo tanto estadísticamente significativa $(\mathrm{p}=0.000)$, con lo cual puede concluirse que existió una diferencia entre las medias de ambos grupos de estudio y con un $95 \%$ de confianza el verdadero valor de la diferencia de medias está contenido en ese intervalo. No se han asumido varianzas iguales, por tanto el $\mathrm{p}$-valor $=0.000<0.05$. En este caso se rechaza la hipótesis nula y se acepta la hipótesis de investigación. Se determina que la variable: La metodología cooperativa con el empleo de tecnologías de la información y la comunicación, mejora significativa para el aprendizaje del idioma inglés.

\section{Conclusiones.}

- Se ha verificado que a través de las TIC se puede innovar significativamente el aprendizaje del idioma inglés contribuyendo al desarrollo de las destrezas comunicativas.

- La prueba estadística demuestra cuantitativamente que la metodología referida mejoró el aprendizaje del idioma inglés en el grupo experimental en relación con el de control en todas las dimensiones estudiadas: escuchar, leer, escribir, hablar y el aprendizaje de la gramática y el vocabulario.

- Los docentes deben actualizar los procesos metodológicos del aprendizaje cooperativo para aplicarlos usando las tecnologías de la información y la comunicación.

- Las metodologías que combinan la cooperación con las TIC favorecen al aprendizaje de las lenguas extranjeras y con ello la posibilidad de ampliar los dominios del conocimiento en otras ciencias de cualquier otra área de estudio, posibilitando a largo plazo contar con un instrumento que permita desarrollar todo el potencial de aprendizaje del estudiante.

\section{Referencias Bibliográficas.}

Barkley, E., \& Cross, P. y. (2007). Técnicas de aprendizaje colaborativo, Barcelona: . Morata. 
Breen, M. ((2014)). Learner contributions to language learning: New directions in research. Routledge.

Castellano, H. (2010). Enseñando con las TIC. Cengage Learning, Buenos Aires.

Hammond, A. (2018). Learning to learn Cooperatively. Forum, 47(4), 18-28.

Hashemi, M. \&. (2013). Application of cooperative learning in EFL classes to enhance the the Students' Language Learning. Procedia - Social and Behavioral Sciences. Masoud Azizinezhad, 138-141.

Johnson, D. (1975). Obtenido de Cooperative learning in the classroom. Obtenido de http://cooperativo.sallep.net/El\%20aprendizaje\%20cooperativo\%20en\%20el\%20au la.pdf

Johnson, D. W. (1999). El aprendizaje cooperativo en elaula. Buenos Aires. Argentina: Paidós.

Johnson, D. W. (2013). Cooperative Learning: Improving University Instruction by Basing Practice On Validated Theory. Journal on Excellence in University Teaching, 26.

Kagan, S. (1994). Cooperative learning. California: San Juan Capristano.

Lin, A. M. (2008). Cambios de paradigma en la enseñanza de inglés como lengua extranjera: el cambio crítico y más allá. Educación y Pedagogía, vol. XX, núm. 51, pp. 11-23.

López, J. (2015). El uso exitoso de la metodología Presentar-Practicar-Producir en el aula estudio de caso. Portafolio programa de TEFL.

M, M. M. (2006). La investigación cualitativa (Síntesis Conceptual . IIPSI, 123-146.

Pérez, M. A. (2004). La integración curricular de los medios y tecnologías de la información y comunicación en la enseñanza de la lengua y la literatura. Quaderns Digitals, , núm. 35, pp. 23-11.

Temprano, A. ( 2012 ). Las TIC en la enseñanza bilingüe, . Ediciones de la U., Bogotá.

Torrego, J. y. (2012). Aprendizaje cooperativo en las aulas. Madrid.

Ur, P. (2005). A course in language teaching. : Cambridge University Press, Nueva York.

Yule, G. (2014). The study of languages, 15a ed. Cambridge University Press.

Zhang, Y. (2010). Cooperative language learning and foreign language learn and teaching. . Journal of language teaching and research. 


\section{PARA CITAR EL ARTÍCULO INDEXADO.}

Inca Chunata, N. M., Reinoso Espinosa, A. G., Macias Silva, E. C., \& Merino Hernández, S. E. (2020). Modelo de aprendizaje cooperativo del idioma inglés utilizando tecnologías de la información y comunicación. ConcienciaDigital, 3(2), 55-68. https://doi.org/10.33262/concienciadigital.v3i2.1208

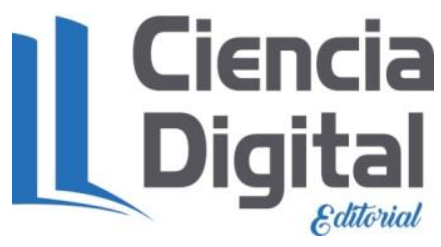

El artículo que se publica es de exclusiva responsabilidad de los autores y no necesariamente reflejan el pensamiento de la Revista Conciencia Digital.

El artículo queda en propiedad de la revista y, por tanto, su publicación parcial y/o total en otro medio tiene que ser autorizado por el director de la Revista Conciencia Digital.

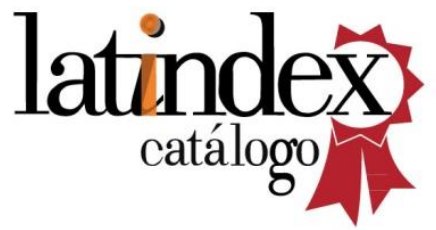

\section{Conciencia}

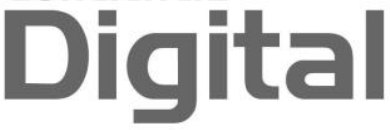

